\title{
Identification of OXA-23-producing Acinetobacter baumannii in Greece, 2010 to 2011
}

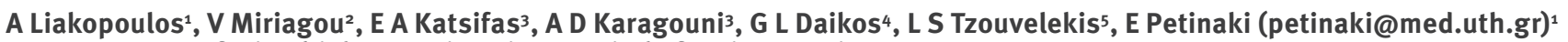

1. Department of Microbiology, University Hospital of Larissa, Larissa, Greece

2. Laboratory of Bacteriology, Hellenic Pasteur Institute, Athens, Greece

3. Faculty of Biology, Department of Botany, Microbiology Group, University of Athens, Athens, Greece

4. 1st Department of Propaedeutic Medicine, Medical School, University of Athens, Athens, Greece

5. Department of Microbiology, Medical School, University of Athens, Athens, Greece

Citation style for this article:

Liakopoulos A, Miriagou V, Katsifas EA, Karagouni AD, Daikos GL, Tzouvelekis LS, Petinaki E. Identification of OXA-23-producing Acinetobacter baumannii in Greece, 2010 to 2011.

Euro Surveill. 2012;17(11):pii=20117. Available online: http://www.eurosurveillance.org/ViewArticle.aspx?Articleld=20117

Article published on 15 March 2012

We report on the sequence type and beta-lactamase content of 174 carbapenem-resistant Acinetobacter baumannii isolates recovered from clinical specimens during 2010 and 2011 in a tertiary care hospital in central Greece. Carbapenem resistance was associated mainly with carriage of the bla ${ }_{\mathrm{OXA}-23}$ gene (in $72.4 \%$ of the isolates). To our knowledge, this is the first description of $A$. baumannii strains producing OXA-23 in Greece. During 2011, in our hospital they rapidly 'replaced' the previously predominant OXA-58positive $A$. baumannii strains.

Previous studies have documented the predominance of oxacillinase (OXA)-58-producers among carbapenem-resistant Acinetobacter baumannii in Greek hospitals [1]. Here, we report the isolation of OXA-23producing $A$. baumannii from clinical specimens taken in 2010 and 2011 at the University Hospital of Larissa in central Greece. To the best of our knowledge, this is the first description of OXA-23-producing A. baumannii in this country.

\section{Background}

A. baumannii has emerged as a leading cause of nosocomial infections, particularly among critically ill patients in intensive care units [2]. A. baumannii clinical isolates are commonly resistant to multiple antimicrobial drug classes and have the ability to survive in the environment for prolonged periods of time, which facilitates their persistence in hospitals [3]. Carbapenems have been widely used to treat infections caused by the microorganism but a trend of increasing resistance to these antibiotics associated with the production of acquired carbapenem-hydrolysing OXA-type class D beta-lactamases has been reported worldwide [4]. Three distinct groups of such acquired beta-lactamases, OXA-23-like, OXA-24/40-like and OXA-58-like, have been described [4].

\section{Collection and analysis of isolates}

The University Hospital of Larissa has 600 beds, with medical, surgical and paediatric subspecialties and one intensive care unit. It serves as one of the main tertiary care hospitals in the district of Thessaly, which has a population of $1,000,000$ inhabitants.

Examination of records kept by the infection control committee of the University Hospital of Larissa showed that carbapenem-resistant $A$. baumannii was isolated more frequently in 2011 than in 2010 (2.1 vs 1.5 cases, respectively, per 1,000 patient-days), while the overall isolation rates of $A$. baumannii remained essentially similar (2.1 and 2.0 cases per 1,000 patientdays, respectively). Furthermore, it was noticed that the carbapenem-resistant $A$. baumannii isolates recovered during 2011 exhibited higher meropenem minimal inhibitory concentrations (MICs), ranging from 128 to $256 \mathrm{mg} / \mathrm{L}$, with both $\mathrm{MIC}_{50}$ and $\mathrm{MIC}_{90}$ values at $256 \mathrm{mg} / \mathrm{L}$. For the isolates from 2010, the range was 16-256 mg/L, with $\mathrm{MIC}_{50}$ and $\mathrm{MIC}_{90}$ values at 24 and 32, respectively). These findings prompted us to explore any changes in the $A$. baumannii population.

We therefore in December 2011, after the annual evaluation of the infection control committee, studied retrospectively single carbapenem-resistant $A$. baumannii clinical isolates (i.e. one per patient) obtained during July to December $2010(n=47)$ and those from January to December $2011(n=127)$. The isolates were recovered from various clinical specimens including, in descending frequency, bronchial secretions, blood, pus and urine. When necessary, species identity was confirmed with VITEK 2 (bioMérieux, France) and/or detection by PCR of bla ${ }_{\text {OXA-51 }}$ the intrinsic carbapenemase gene of $A$. baumannii [5]. MICs of various antimicrobials, including imipenem and meropenem, were re-determined by the Etest (bioMérieux, Sweden). Susceptibility status was defined according to the latest European Committee on Antimicrobial Susceptibility Testing breakpoints [6]. 
All 174 isolates were typed by three loci-sequencing typing [5,7]; allele numbers and sequence types were assigned based on the information in the respective databases. Identification of CHDL-encoding genes and mapping of their genetic environment were performed by PCR assays and sequencing of the respective amplicons, as described elsewhere $[8,9]$.

\section{Characteristics of the carbapenem-} resistant $A$. baumannii population

Of the 174 carbapenem-resistant $A$. baumannii isolates, $125(71.8 \%)$ carried bla $a_{\mathrm{OXA}-23}, 48(27.6 \%)$ carried $b l a_{0 \times A-58}$ and one (0.6\%) was positive for both genes. The majority of bla $a_{\mathrm{O} A-58}$ carriers $(\mathrm{n}=42)$ were isolated during 2010. Five of the bla $a_{\mathrm{OXA}-23}$ carriers were isolated in late 2010 from patients in the intensive care unit. The first two were isolated from patients in October 2010. The first patient was in their midzos and the second in their early 70s. Both patients had no previous medical history of underlying disease such as diabetes, cancer or immunosuppression before being hospitalised. These two patients had been treated for at least two weeks before the isolates were cultured and their infections (both bacteraemia) were considered as hospital acquired, as was the case for the remaining patients infected with OXA-23-producing A. baumannii. Medical records of the first two patients did not indicate prior hospitalisation in the past six months or any travel abroad. It is therefore possible that OXA23-positive $A$. baumannii had already circulated in the hospital.

Environmental screening was not carried out. We cannot provide reliable hypotheses regarding potential routes of introduction of OXA-23 producers to the hospital. Among the carbapenem-resistant $A$. baumannii isolates detected in 2011, the bla ${ }_{\mathrm{OXA}-23}$ carriers predominated (120/127; 95\%): 43 were isolated from patients in the intensive care unit and the rest from various medical $(n=60)$ and surgical wards $(n=17)$. Of the other seven isolates, recovered in a sporadic fashion throughout 2011, six were positive for $b a_{0 \times A-58}$ and one carried both $b a_{\mathrm{OXA}-58}$ and $b l a_{\mathrm{OXA}-23}$. According to the three loci-sequencing typing, 24 bla $a_{\text {OXA-58 }}$-positive isolates belonged to sequence type (ST) 106, 23 to ST201 and one exhibited a novel allelic profile (2-1-2). ST101 was prevalent among bla $a_{\text {OXA-23 }}$ carriers $(n=101)$, the remaining 24 isolates being classified as ST201. The isolate harbouring both carbapenemase genes belonged to ST106. The isolation frequency of the isolates is shown in the Figure.

Apart from resistance to at least one carbapenem (imipenem and/or meropenem), all 174 isolates exhibited resistance to multiple drugs including penicillin-inhibitor combinations, oxyimino-cephalosporins, aminoglycosides and fluoroquinolones. All were susceptible to colistin. The bla $a_{\mathrm{OXA-23}}$-positive isolates were generally inhibited by higher concentrations of meropenem as compared with bla $a_{\mathrm{OXA}-58}$ carriers. This is consistent with previous findings indicating that OXA-23 confers to
A. baumannii higher levels of carbapenem resistance, as compared with OXA-58, although both enzymes exhibit relatively weak carbapenemase activities [10]. The genetic environment of $b a_{0 \times A-23}$ gene was investigated in 20 isolates representing all sequence types and beta-lactamase combinations found. In all cases, bla $a_{\text {OXA-23 }}$ occurred as part of the previously characterised Tn2006 transposon bracketed by two ISAba1 (insertion sequences) in opposite orientation [9].

\section{Discussion}

During 1999 to 2009, A. baumannii strains carrying the bla ${ }_{\mathrm{OXA}-58}$ carbapenemase gene predominated among carbapenem-resistant isolates of this species in the hospital flora in various Mediterranean countries including Italy, Greece, Lebanon and Turkey $[1,11]$. Since 2009 , isolation of $A$. baumannii producing the OXA-23 carbapenemase has been increasingly reported in European countries [12-14]. Of note, a massive 'replacement' of OXA-58 A. baumannii by OXA-23 producers, similar to that observed here, has recently been described in Italian hospitals [15]. It was hypothesised that the higher carbapenem MICs of the $b a_{\mathrm{OXA}-23_{3}}{ }^{-}$ positive isolates may provide a selective advantage in the hospital setting. Yet, the change of the A. baumannii population seen in our study was too abrupt to be explained by the operation of carbapenem selective pressure alone. Whatever the reasons for the shift in

\section{FIGURE}

Isolation frequencies of carbapenem-resistant Acinetobacter baumannii strains, University Hospital of Larissa, Thessaly, Greece, July 2010-December 2011 $(n=174)$

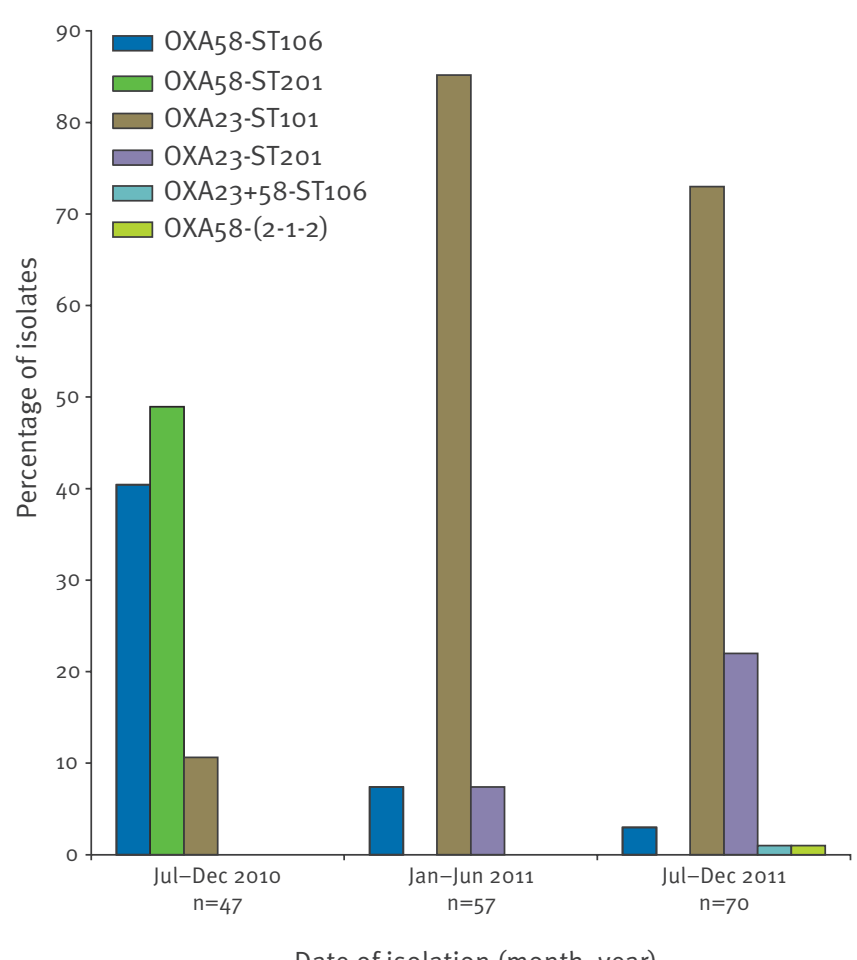

ST: sequence type. 
the $A$. baumannii population, the spread of strains producing OXA-23 seems to reflect a global trend towards a predominance of $b l a_{0 \times A-23}$ carriers belonging to the international clonal lineages I and II [16,17]. Spread of multidrug-resistant strains producing OXA-23 may aggravate the therapeutic problems caused by this species by further limiting treatment options.

It is highly likely that similar changes have also occurred in the $A$. baumannii population in tertiary care hospitals in the major urban centres of Greece, Athens and Thessaloniki. The available resources however, have been almost exclusively allocated to detecting and containing carbapenemase-producing Enterobacteriaceae. Yet, we believe that a low-cost study involving a limited number of sentinel hospitals would provide a comprehensive picture regarding the A. baumannii strains circulating in this country.

Hospital personnel have been informed about our findings and infection control measures have been reinforced.

\section{Acknowledgments}

This work was partially supported by the Medical School of the University of Thessalia (Grant No. 3917) and the Hellenic Pasteur Institute, Athens, Greece. We thank A. Papaioannou for technical assistance. countries with a multilocus sequence typing scheme. Clin Microbiol Infect. 2011;17(2):197-201.

12. Brigante G, Migliavacca R, Bramati S, Motta E, Nucleo E, Manenti $M$, et al. Emergence and spread of a multidrugresistant Acinetobacter baumannii clone producing both the OXA-23 carbapenemase and the $16 \mathrm{~S}$ rRNA methylase ArmA. J Med Microbiol. 2012 Jan 26. [Epub ahead of print].

13. Karah N, Giske CG, Sundsfjord A, Samuelsen $\emptyset$. A diversity of OXA-carbapenemases and class 1 integrons among carbapenem-resistant Acinetobacter baumannii clinical isolates from Sweden belonging to different international clonal lineages. Microb Drug Resist. 2011;17(4):545-9.

14. Bonnin RA, Poirel L, Licker M, Nordmann P. Genetic diversity of carbapenem-hydrolysing $\beta$-lactamases in Acinetobacter baumannii from Romanian hospitals. Clin Microbiol Infect. 2011;17(10):1524-8.

15. D’Arezzo S, Principe L, Capone A, Petrosillo N, Petrucca A Visca P. Changing carbapenemase gene pattern in an epidemic multidrug-resistant Acinetobacter baumannii lineage causing multiple outbreaks in central Italy. J Antimicrob Chemother. 2011;66(1):54-61.

16. Higgins PG, Dammhayn C, Hackel M, Seifert H. Global spread of carbapenem-resistant Acinetobacter baumannii. J Antimicrob Chemother. 2010;65(2):233-8.

17. Mugnier PD, Poirel L, Naas T, Nordmann P. Worldwide dissemination of the blaOXA-23 carbapenemase gene of Acinetobacter baumannii. Emerg Infect Dis. 2010;16(1):35-40.

References

1. Gogou V, Pournaras S, Giannouli M, Voulgari E, Piperaki ET, Zarrilli R, et al. Evolution of multidrug-resistant Acinetobacter baumannii clonal lineages: a 10 year study in Greece (200009). J Antimicrob Chemother. 2011;66(12):2767-72.

2. Chastre J. Infections due to Acinetobacter baumannii in the ICU. Semin Respir Crit Care Med. 2003;24(1):69-78.

3. Neonakis IK, Spandidos DA, Petinaki E. Confronting multidrugresistant Acinetobacter baumannii: a review. Int J Antimicrob Agents. 2011;37(2):102-9.

4. Poirel L, Nordmann P. Carbapenem resistance in Acinetobacter baumannii: mechanisms and epidemiology. Clin Microbiol Infect. 2006;12(9):826-36.

5. Turton JF, Gabriel SN, Valderrey C, Kaufmann ME, Pitt TL. Use of sequence-based typing and multiplex PCR to identify clonal lineages of outbreak strains of Acinetobacter baumannii. Clin Microbiol Infect. 2007;13(8):807-15.

6. European Committee on Antimicrobial Susceptibility Testing (EUCAST). Breakpoint tables for interpretation of MICS and zone diameters. 1 Jan 2012. Available from: http://www.eucast. org/fileadmin/src/media/PDFs/EUCAST_files/Breakpoint tables/Breakpoint table v 2.0 120221.pd

7. Health Protection Agency (HPA). Acinetobacter baumannii sequence typing home page. London: HPA. [Accessed 1 Jan 2012]. Available from: http://www.hpa-bioinformatics.org.uk/ $\mathrm{AB} /$ home.php

8. Woodford N, Ellington MI, Coelho JM, Turton JF, Ward ME, Brown S, et al. Multiplex PCR for genes encoding prevalent OXA carbapenemases in Acinetobacter spp. Int J Antimicrob Agents. 2006;27(4):351-3.

9. Corvec S, Poirel L, Naas T, Drugeon H, Nordmann P. Genetics and expression of the carbapenem-hydrolyzing oxacillinase gene blaOXA-23 in Acinetobacter baumannii. Antimicrob Agents Chemother. 2007;51(4):1530-3.

10. Héritier C, Poirel L, Lambert T, Nordmann P. Contribution of acquired carbapenem-hydrolyzing oxacillinases to carbapenem resistance in Acinetobacter baumannii. Antimicrob Agents Chemother. 2005;49(8):3198-202.

11. Di Popolo A, Giannouli M, Triassi M, Brisse S, Zarrilli R. Molecular epidemiological investigation of multidrug-resistant Acinetobacter baumannii strains in four Mediterranean 